\title{
Integración de la competencia transversal de "conocimiento de problemas contemporáneos" en la asignatura Vibraciones Mecánicas
}

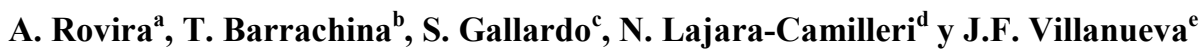
${ }^{a}$ CIIM. Dpto. de Ingerniería Mecánica y de Materiales. Universitat Politècnica de València. arovira@mcm.upv.es, ${ }^{b}$ Dpto. de Ingeniería Química y Nuclear. Universitat Politècnica de València. tbarrachina@iqn.upv.es, ${ }^{\mathrm{c} D p t o . ~ d e ~ I n g e n i e r i ́ a ~ Q u i ́ m i c a ~ y ~ N u c l e a r . ~ U n i v e r s i t a t ~ P o l i t e ̀ c n i c a ~ d e ~ V a l e ̀ n c i a . ~}$ sergalbe@iqn.upv.es, ${ }^{\mathrm{d}}$ CEGEA. Dpto. de Economía y Ciencias Sociales. Universitat Politècnica de València. nalade@cegea.upv.es y ${ }^{\mathrm{e}}$ Dpto. de Estadística e Investigación Operativa Aplicadas y Calidad. Universitat Politècnica de València. jovillo0@upvnet.upv.es
\end{abstract}

\begin{abstract}
Current degrees belonging to the European Higher Education Area (EHEA) represent a change on the learning towards the competence based education. After the implantation of the new degree programs and in order to group different skills profiles, the Universitat Politecnica de Valencia (UPV) defined a list of generic competences. This list consists of a total of 13 competences to be trained and assessed in different subjects forming part of a degree.

One of these competences is the "knowledge of contemporary issues", which refers to the need for students to understand the political, social, legal and environmental issues and values, as defined by the UPV. This competence also takes into account the mechanism of expansion and dissemination of knowledge.

In this paper a preliminary experience carried out in the Mechanical Vibrations course is described. An activity framed in a laboratory session has been designed to this end, where this competence is introduced related to the contents of the subject. The results of this experience indicate that the training of the competence "knowledge of contemporary issues" contributes to improve learning in the subject.
\end{abstract}

Keywords: competences, training and assessment, UPV transversal competences, "knowledge of contenporary issues"

\footnotetext{
Resumen

Los actuales planes de estudios pertenecientes al Espacio Europeo de Educación Superior (EEES) representan un cambio en el aprendizaje hacia la educación basada en competencias. Tras la implantación de los nuevos programas de grado y con el fin de agrupar los distintos perfiles competenciales, la Universitat Politècnica de València (UPV) ha definido una lista de competencias transversales. Esta lista se compone de un total de
} 
Integración de la competencia transversal de "conocimiento de problemas contemporáneos" en la asignatura Vibraciones Mecánicas

13 competencias para ser trabajadas y evaluadas en diferentes asignaturas que forman parte cada titulación.

Una de estas competencias es el "conocimiento de problemas contemporáneos", que se refiere a la necesidad de que los estudiantes comprendan los problemas y valores políticos, sociales, legales y ambientales, tal como se define por la UPV. Esta competencia también tiene en cuenta el mecanismo de expansión y difusión del conocimiento.

En este trabajo se describe una experiencia preliminar llevada a cabo en la asignatura Vibraciones Mecánicas. Para ello se ha diseñado un actividad, enmarcada en una sesión de prácticas de laboratorio, en la que se introduce esta dimensión relacionada con los contenidos de la asignatura. Los resultados de esta experiencia indican que el trabajo de la competencia "conocimiento de problemas contemporáneos" contribuye a mejorar el aprendizaje en la asignatura.

Palabras clave: competencias, trabajo y evaluación, competencias transversales UPV, "conocimiento de problemas contemporáneos"

\section{Introducción}

Los actuales planes de estudio del Espacio Europeo de Educación Superior (EEES) representan un cambio de enfoque desde la formación en contenidos a la formación en competencias, tanto específicas como transversales o genéricas.

A través del proceso de acreditación de la comisión Accreditation Board for Engineering and Technology (ABET) llevado a cabo en algunas titulaciones en la Universitat Politècnica de València (UPV), quedó patente la necesidad de evaluar y de obtener evidencias de las competencias, principalmente transversales, que deben trabajarse en las titulaciones en cuestión.

En este contexto, y con el objeto de agrupar las distintas competencias transversales que conforman las distintas titulaciones impartidas en la UPV, se definieron 13 competencias transversales comunes a todas las titulaciones. De este forma se pueden diseñar procesos de evaluación y acreditación flexibles e innovadores así como implementar procesos de evaluación y acreditación en todos los títulos oficiales. El objetivo es incorporar estas competencias a la formación de los estudiantes e incluir los grados o niveles de adquisición de estas competencias en los currículum de los egresados con el fin de dar visibilidad de los resultados de aprendizaje adquiridos. Para alcanzar este objetivo se requiere rediseñar el enfoque y la metodología de las asignaturas para incorporar las distintas competencias transversales.

En este estudio se ha tomado la competencia transversal "conocimiento de problemas contemporáneos" (CT-10). Se trata de una dimensión que, si bien de forma habitual es incluida en las aulas, normalmente ha sido el profesor el encargado de aportarla mediante

(cc) EY-NC-ND 2016, Universitat Politècnica de València 
ejemplos o estudios de caso. La novedad y el reto que se plantean actualmente es trasladar esta cuestión al estudiante para que desarrolle y evolucione esta capacidad.

En el presente trabajo se presenta una experiencia realizada en la asignatura Vibraciones Mecánicas, con el objeto de integrar la competencia transversal de "conocimiento de problemas contemporáneos".

Este trabajo se enmarca dentro de un Proyecto de Innovación y Mejora Educativa (PIME) titulado Desarrollo y evaluación de la competencia transversal de "conocimiento de problemas contemporáneos".

\section{Objetivos}

Los objetivos de este trabajo son dos:

- Integrar la competencia de "conocimiento de problemas contemporáneos" en asignaturas orientadas a la resolución de problemas en el ámbito de la ingeniería.

- Presentar la experiencia realizada en la asignatura Vibraciones Mecánicas.

\section{La asignatura Vibraciones Mecánicas}

La asignatura Vibraciones Mecánicas forma parte del $4^{\circ}$ curso del Grado en Ingeniería Mecánica (GIM) impartido en la Escuela Técnica Superior de Ingeniería del Diseño (ETSID) de la Universitat Politècnica de València (UPV). Tiene un total de 4,5 créditos distribuidos en un $50 \%$ en clases de teoría y un $50 \%$ en clases de prácticas (ver Tabla 1 ).

Tabla 1. Datos de la asignatura Vibraciones Mecánicas

\begin{tabular}{|l|l|l|}
\hline Código: 12577 & Asignatura: Vibraciones Mecánicas \\
\hline Créditos totales: 4,5 & Créditos teoría: 2,25 & Créditos de prácticas: 2,25 \\
\hline Tipo: Obligatorio & $\begin{array}{l}\text { Módulo: Especialidad } \\
\text { Mecánica }\end{array}$ & $\begin{array}{l}\text { Materia: Ingeniería } \\
\text { Mecánica y de Materiales II }\end{array}$ \\
\hline $\begin{array}{l}\text { Titulación: Grado en } \\
\text { Ingeniería Mecánica (GIM) }\end{array}$ & $\begin{array}{l}\text { Escuela: Escuela Técnica Superior de Ingeniería del Diseño } \\
\text { (ETSID) }\end{array}$ \\
\hline
\end{tabular}

Esta asignatura está enfocada al modelado y análisis de problemas relacionados con las vibraciones mecánicas desde un punto de vista práctico. Además, se trata de una asignatura de último curso de la titulación, que se sirve de los conocimientos y competencias de las asignaturas precedentes, con el fin de resolver problemas en el ámbito de la ingeniería mecánica.

Debido a la complejidad tanto de la asignatura como del propio entorno industrial, resulta imposible resolver todos los tipos de problemas que puedan aparecen en la vida profesional; más aún, si se tiene en cuenta que en casos reales los problemas no se encuentran perfectamente definidos ni acotados dentro de los temas desarrollados en la asignatura. Por

(cc) EY-NC-ND 2016, Universitat Politècnica de València

Congreso In-Red (2016) 
Integración de la competencia transversal de "conocimiento de problemas contemporáneos" en la asignatura Vibraciones Mecánicas

tanto, resulta interesante ofrecer un marco más amplio con el fin de incentivar la expansión y difusión del conocimiento.

El trabajo de la competencia de "conocimiento de problemas contemporáneos" se ha planteado con el objeto de ofrecer una visión global a los problemas derivados de la ingeniería y que afectan a la sociedad.

\section{Desarrollo de la innovación}

\subsection{La competencia de conocimiento de problemas contemporáneos}

La competencia de "conocimiento de problemas contemporáneos" hace referencia a la necesidad de que los estudiantes comprendan las cuestiones y valores políticos, sociales, legales y medioambientales contemporáneos, así como los mecanismos de expasión y difusión del conocimiento. Según esta competencia, los alumnos deben desarrollar la capacidad de "estar al día" de los eventos actuales en su campo de conocimiento y en la sociedad en general (UPV, 2015a). Es una componente fundamental para los futuros profesionales ya que les permite contextualizar los conocimientos y aplicarlos de forma práctica. Además, facilita la reflexión y el desarrollo de una posición crítica razonada.

Siguiendo la clasificación del Proyecto Tuning (2014), se trata de una competencia de tipo sistémico, ya que a pesar de que resulta básica para la adquisición de otras competencias y otros aprendizajes y afecta al conocimiento general básico -y por ello podría ser considerada instrumental-, en realidad se trata de una capacidad de visión y análisis de realidades multidimensionales, más cercano al aprendizaje autónomo y al conocimientos de otras culturas y costumbres.

Resulta difícil encontrar bibliografía especializada que haya abordado previamente la contextualización de esta competencia ya que en muchas ocasiones se considera incluida dentro del aprendizaje permanente (Villa, 2007), en la capacidad para detectar nuevas oportunidades (ANECA 2007) o en la capacidad de gestionar la información y el conocimiento de su ámbito disciplinar (UM, 2008).

Villa (2003) señala que la sociedad demanda a la Universidad no sólo profesionales bien formados sino que estén preparados para desenvolverse como buenos ciudadanos. Las dimensiones que esta cuestión entraña, implican también el conocimiento actual de la actualidad en relación a su sector, la reflexión y la valoración.

Para definir el nivel a desarrollar las competencias transversales, en la UPV se han definido tres niveles. Un primer nivel para asignaturas de $1^{\mathrm{er}}$ y $2^{\circ}$ curso de grado; un segundo nivel para $3^{\circ}$ y $4^{\circ}$ de grado; y un tercer nivel para máster.

Para el caso de la competencia de "conocimiento de problemas contemporáneos", el primer nivel de dominio busca reconocer los problemas contemporáneos que afectan al campo profesional; el segundo nivel, analizar estos problemas, y, el tercer nivel, proponer soluciones, evaluar estas soluciones y evaluar sus consecuencias.

(cc) EY-NC-ND 2016, Universitat Politècnica de València

Congreso IN-RED (2016) 


\subsection{Trabajo y evaluación de la competencia}

Según Leiva-Brondo (2015), el profesorado se enfrenta a la necesidad de identificar métodos adecuados para el trabajo de la competencia, así como para la posterior evaluación del grado de adquisición por parte de los estudiantes.

En la bibliografía se presentan distintas alternativas como el estudio del caso (Schwartz, 2012), el debate (Balachandran, 2008) o la combinación con trabajos académicos (Sala et al., 2011). El objetivo es estimular a los alumnos para que relacionen distintos conceptos de las asignaturas con "problemas contemporáneos".

Considerando los tres niveles de trabajo y evaluación de las competencias transversales, adoptado por la UPV (2015b), para el segundo nivel, correspondiente a la asignatura Vibraciones Mecánicas, se proponen cinco indicadores: identificar las causas que han dado lugar a la situación actual, identificar las consecuencias del ámbito del problema en el campo profesional, distinguir las partes que componen el problema, hacer un razonamiento crítico de las soluciones ya propuestas y proponer nuevas soluciones. De esta forma la evaluación del grado de adquisición de la competencia se puede hacer a través de una tabla de evaluación (rúbrica) o a través de una lista de control.

\section{Experiencia en la asignatura Vibraciones Mecánicas}

El objetivo de esta experiencia es integrar la competencia de "conocimiento de problemas contemporáneos" en la asignatura Vibraciones Mecánicas. Inicialmente representaba un reto para los profesores debido a la naturaleza de la asignatura, esencialmente computacional y relacionada con el modelado y análisis de problemas. Sin embargo, la introducción de esta actividad persigue ampliar el horizonte de la asignatura con el fin de visualizar problemas mayores y analizarlos desde un punto de vista conceptual. Este distinto enfoque ayuda a comprender la asignatura y tal como dice Leiva-Brondo (2015), consigue involucrar más a los alumnos en el proceso de enseñanza aprendizaje.

La actividad se ha diseñado para que se realice en una sesión de prácticas de laboratorio, con una duración de dos horas. Para el trabajo de la competencia se visualiza un vídeo y se analiza éste usando la metodología del caso. El análisis está guiado por el profesor y persigue cubrir los indicadores señalados en el apartado 4.2. En esta actividad se hace hincapié en la importancia de conocer no sólo aspectos técnicos, sino de valorar la relación de la asignatura con la realidad profesional y su influencia en la sociedad.

El vídeo escogido muestra un problema que presentó el Puente del Milenio en Londres, tras su inauguración (Fig. 1). Se trata de un puente peatonal, en el que, debido a la excitación sincronizada de los peatones, se produce una vibración en el plano horizontal que causa que sea difícil andar y mantener el equilibrio.

(cc) EY-NC-ND 2016, Universitat Politècnica de València

Congreso In-Red (2016) 


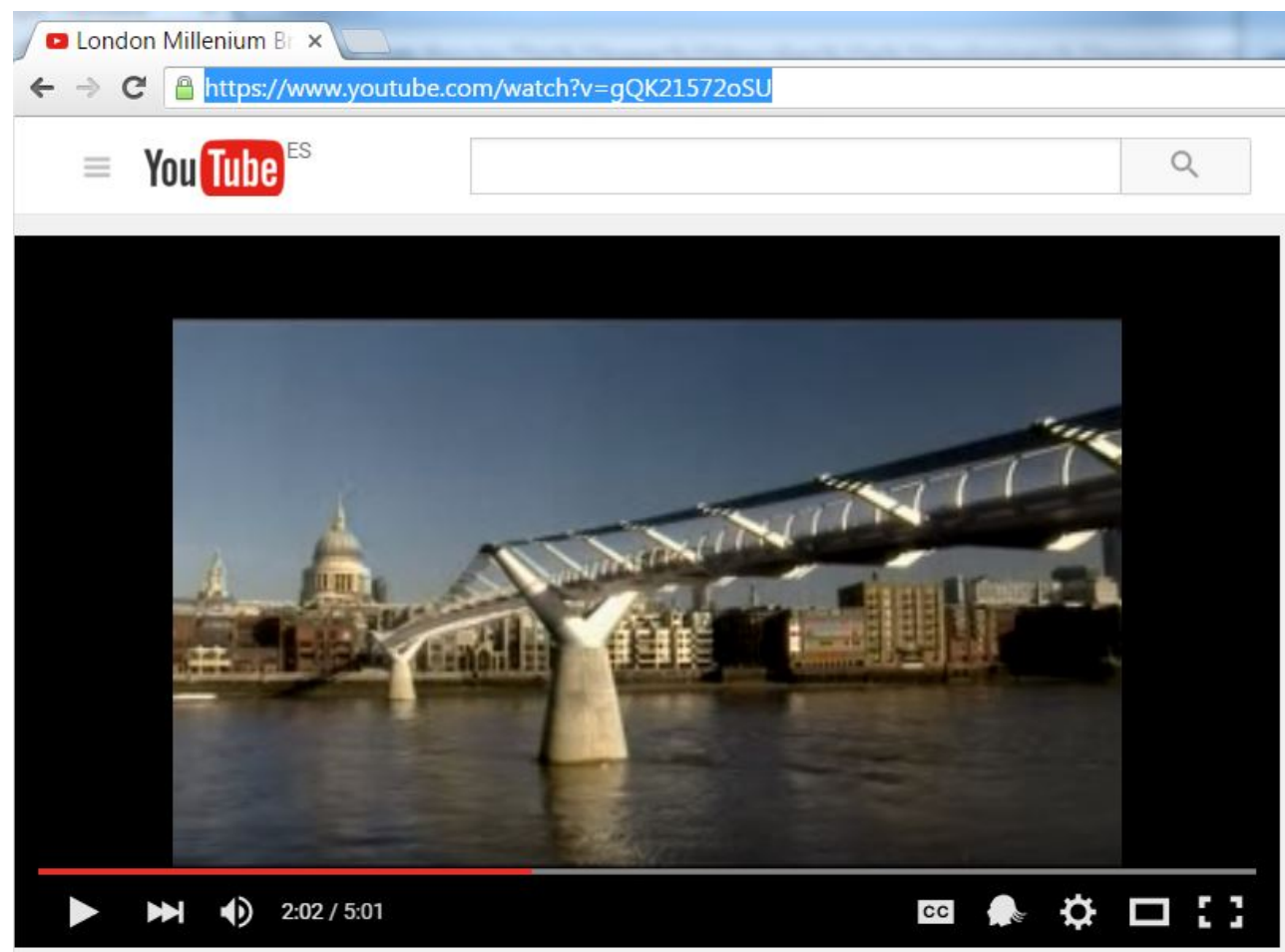

\section{London Millenium Bridge Opening}

Fig. 1 Captura de pantalla del vídeo visualizadoen clase (Youtube, 2015).

El caso presentado es un claro ejemplo de un mal diseño ingenieril que provoca problemas no sólo técnicos, sino también éticos y socioeconómicos. Durante la resolución, se plantea resolver el compromiso que aparece muchas veces entre la estética y la funcionalidad; dilema que normalmente no aparece en la formación de los ingenieros. Cabe destacar que no se pretende resolver el problema, puesto que es un asunto complejo de resolver con los contenidos de la asignatura, sin embargo, sí que se analizan diversas soluciones y se consensúa una posible solución. Finalmente, se plantea la relación con otros problemas contemporáneos, como son la corrupción o la ética.

Esta actividad se realizó para un grupo de prácticas de laboratorio, compuesto por 20 estudiantes. Se realizó una evaluación global del grado de adquisición de la competencia. Tras acabar la actividad, se pasó una encuesta anónima con el fin de evaluar la integración de la competencia en la asignatura y disponer de un feedback de la opinión de los alumnos para futuras mejoras.

\section{Resultados}

\subsection{Evaluación del grado de adquisición de la competencia para el grupo}

La Tabla 2 muestra la rúbrica empleada para la evaluación del grado de adquisición de la competencia de "conocimiento de problemas contemporáneos". Esta rúbrica es la propuesta por la UPV para las asignatura de segundo nivel (UPV, 2015b).

(cc) EY-NC-ND 2016, Universitat Politècnica de València

Congreso IN-RED (2016) 


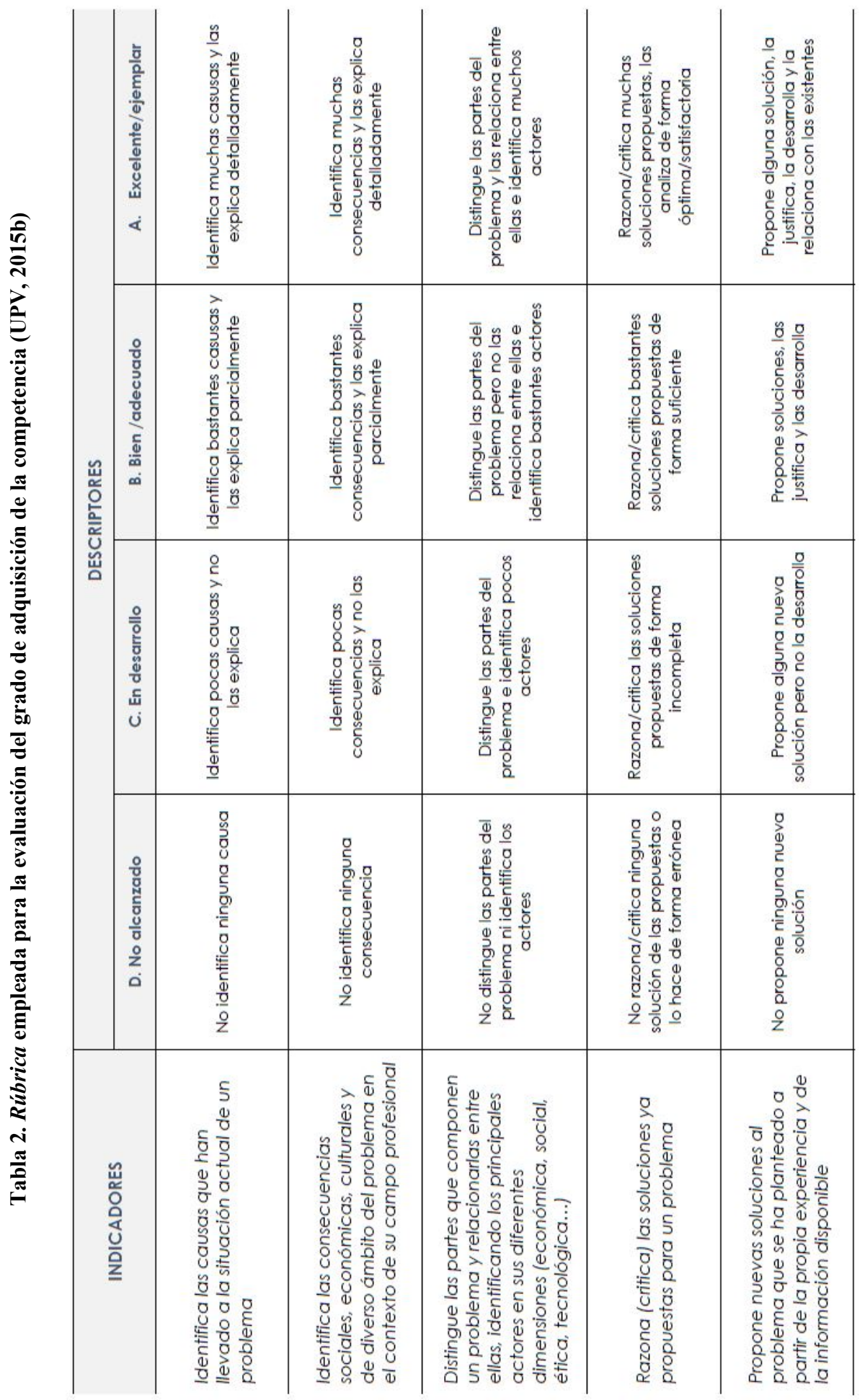

(cc) BY-NC-ND 2016, Universitat Politècnica de València 
Integración de la competencia transversal de "conocimiento de problemas contemporáneos" en la asignatura Vibraciones Mecánicas

La Fig. 2 muestra el resultado de la evaluación del grado de adquisición de la competencia para el grupo, según la rúbrica empleada. La actividad se realiza usando la metodología del caso para todo el grupo de alumnos de la sesión de prácticas. Según los descriptores de la rúbrica de la Tabla 2 se concluye que el grupo:

- identifica bastantes causas y las explica parcialmente,

- identifica bastantes consecuencias y las explica parcialmente,

- distingue las partes del problema y las relaciona entre ellas e identifica muchos actores,

- razona muchas soluciones propuestas y las analiza de forma satisfactoria y

- propone soluciones, las justifica y las desarrolla.

La media obtenida en los cuatro indicadores (GLOBAL) es de 3,4, que se redondearía a la categoría "B. Bien / adecuado".

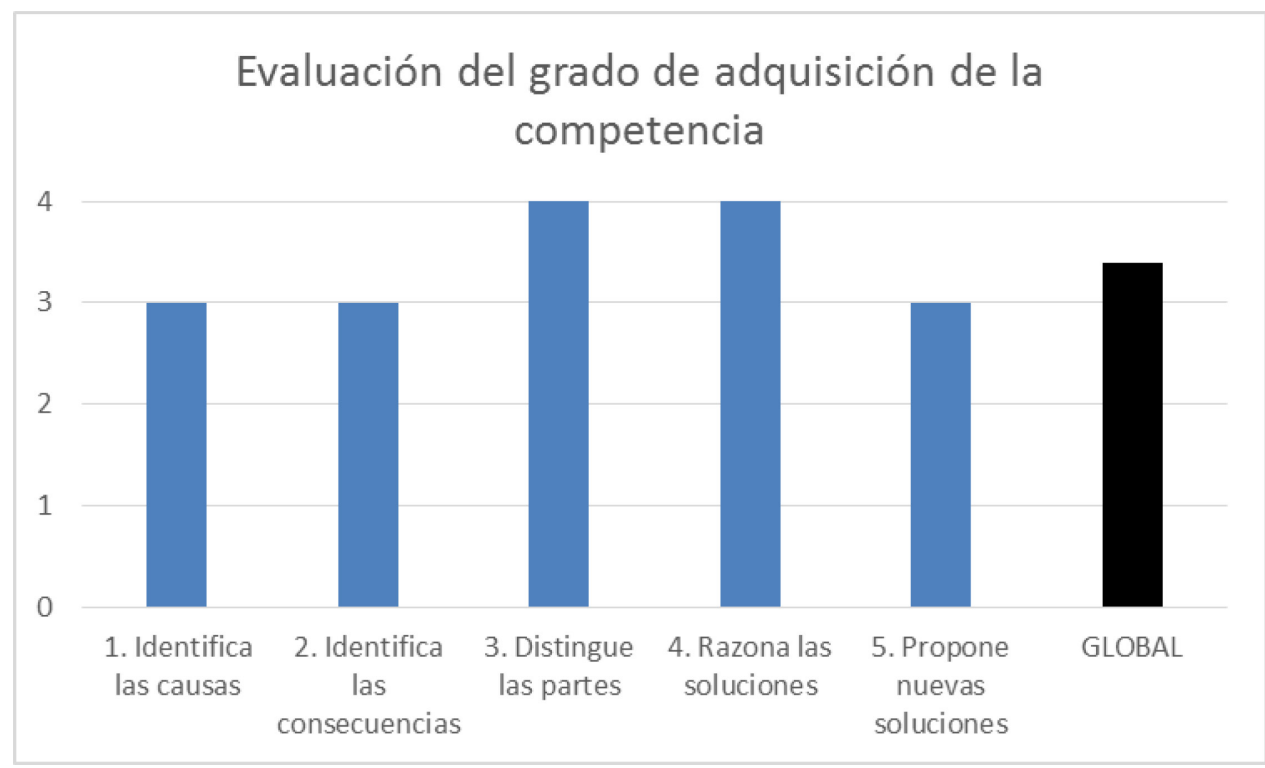

Fig. 2 Evaluación del gado de adquisición de la competencia, donde 0 se corresponde con "opción no contestada", 1 con "D-no alcanzado", 2 con "C-en desarrollo", 3 con "B-bien / adecuado" y 4 con "A-Excelente / ejemplar”, según la escala utilizada en la rúbrica de la Tabla 2.

\subsection{Evaluación de la integración de la competencia en la asignatura}

En este apartado se presentan los resultados de la evaluación realizada por los alumnos a través de un cuestionario. El cuestionario se compone de cuatro preguntas (ver Tabla 3) y se emplea una escala tipo Likert de cinco niveles, donde 1 se corresponde con totalmente en desacuerdo (TD), 2, más bien en desacuerdo (MBD), 3, término medio (TM), 4, más bien de acuerdo (MBA) y 5, totalmente de acuerdo (TA). 
Tabla 3. Cuestionario para la evaluación de la actividad

\begin{tabular}{|c|c|c|c|c|c|}
\hline & 1 & 2 & 3 & 4 & 5 \\
\hline $\begin{array}{l}\text { 1. La asignatura Vibraciones Mecánicas tiene un elevado contenido teórico y, en } \\
\text { muchos casos, es difícil ver la aplicabilidad en el campo de la ingeniería. }\end{array}$ & & & & & \\
\hline $\begin{array}{l}\text { 2. El hecho de presentar ejemplos de problemas contemporáneos, relacionados } \\
\text { con la asignatura, potencia el aprendizaje de ésta. }\end{array}$ & & & & & \\
\hline $\begin{array}{l}\text { 3. Un mal diseño de un componente o estructura es un problema técnico, ético y } \\
\text { socioeconómico. }\end{array}$ & & & & & \\
\hline $\begin{array}{l}\text { 4. Los contenidos de la asignatura ayudan a resolver problemas como los } \\
\text { presentados en la sesión de prácticas. }\end{array}$ & & & & & \\
\hline
\end{tabular}

La primera pregunta es una pregunta introductoria con el objeto de diagnosticar si se está conectando con los alumnos, o quizá sea necesario introducir más ejemplos o aplicaciones en los ejercicios de la asignatura. La segunda pregunta está muy relacionada con la anterior, pero ya se introduce la idea de "problemas contemporáneos" que hacen referencia a cuestiones cuya resolución queda fuera del alcance de la asignatura. La tercera pregunta pretende hacer reflexionar a los alumnos sobre las consecuencias, no solo técnicas, de un mal diseño en ingeniería. Finalmente, la cuarta pregunta es un indicador de la comprensión de la asignatura.

Las Figs. 3-6 muestran las respuesta de los alumnos al cuestionario de la Tabla 3. El grupo está formado por 20 alumnos.

Analizando las respuestas a la primera pregunta, los alumnos están, en general, en desacuerdo con que la asignatura tiene un contenido elevado teórico y que es difícil ver la aplicabilidad. Este resultado indica que la asignatura está bien planteada ya que aunque la asignatura tiene una elevada complejidad, no se pierde su aplicación.

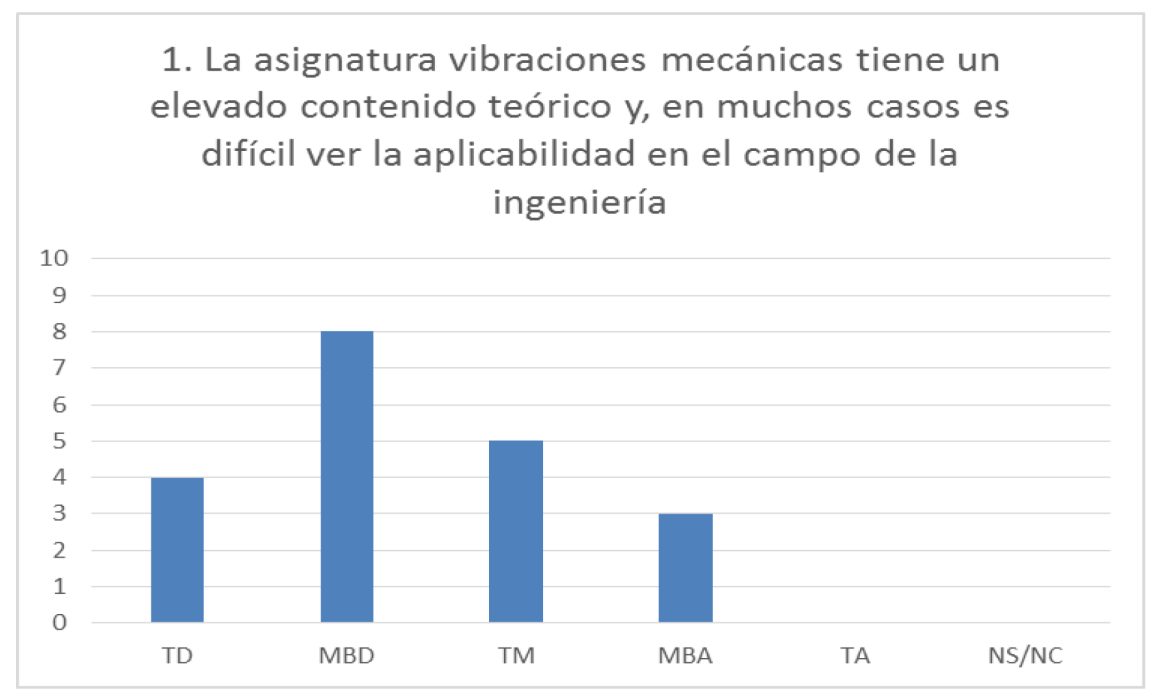

Fig. 3 Respuestas a la primera pregunta del cuestionario de la Tabla 3. 
Integración de la competencia transversal de "conocimiento de problemas contemporáneos" en la asignatura Vibraciones Mecánicas

Las preguntas 2, 3 y 4 están directamente relacionadas con la actividad; un grado de acuerdo alto de los alumnos con las afirmaciones presentadas indicará que la actividad es adecuada para la integración de la competencia transversal en la asignatura. Así lo demuestran las respuestas de los alumnos, ya que agrupando las respuestas que están más bien de acuerdo (MBA) o totalmente de acuerdo (TA), se obtienen 18 respestas sobre 20 en la pregunta 2, 19/20 en la pregunta 3 y 20/20 en la pregunta 4. Estos resultados indican que la actividad realizada no solo sirve para el trabajo de la competencia de "conocimiento de problemas contemporáneos" sino que potencia el trabajo y el aprendizaje de la asignatura.

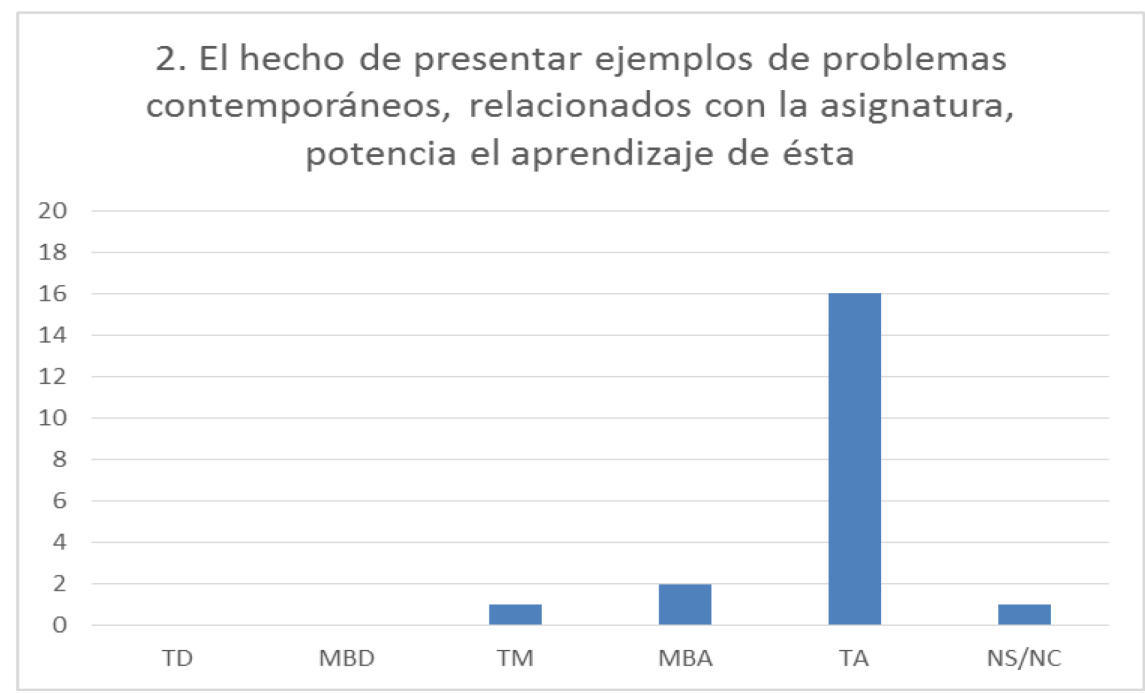

Fig. 4 Respuestas a la segunda pregunta del cuestionario de la Tabla 3.

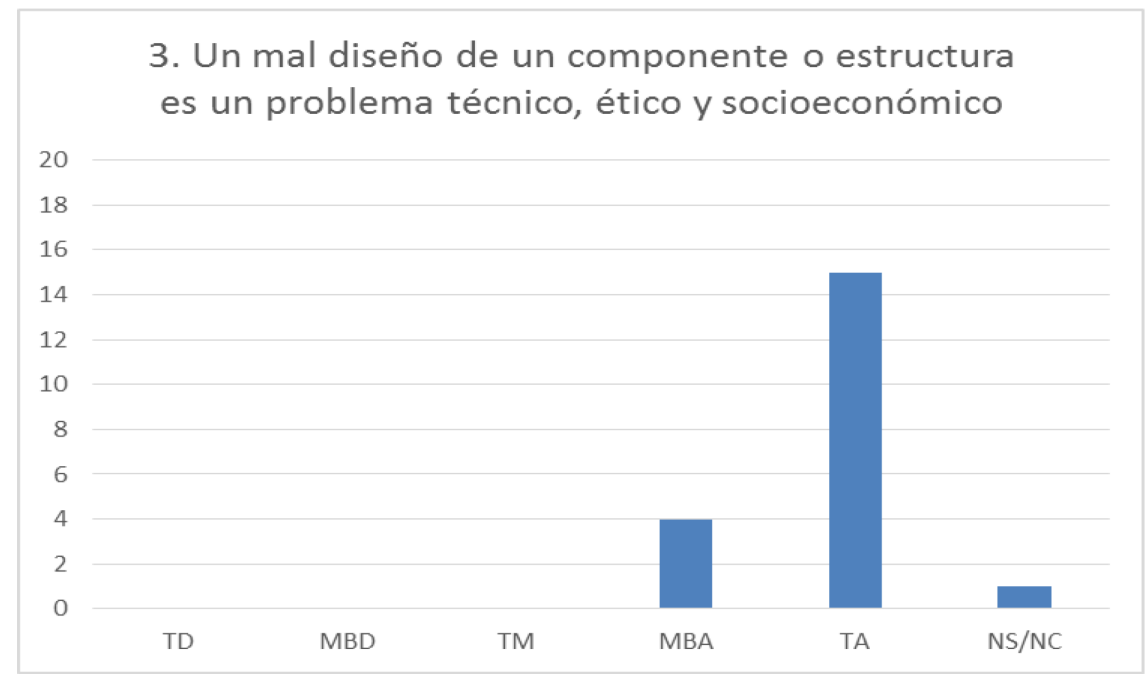

Fig. 5 Respuestas a la tercera pregunta del cuestionario de la Tabla 3.

(cc)) EY-NC-ND 2016, Universitat Politècnica de València 


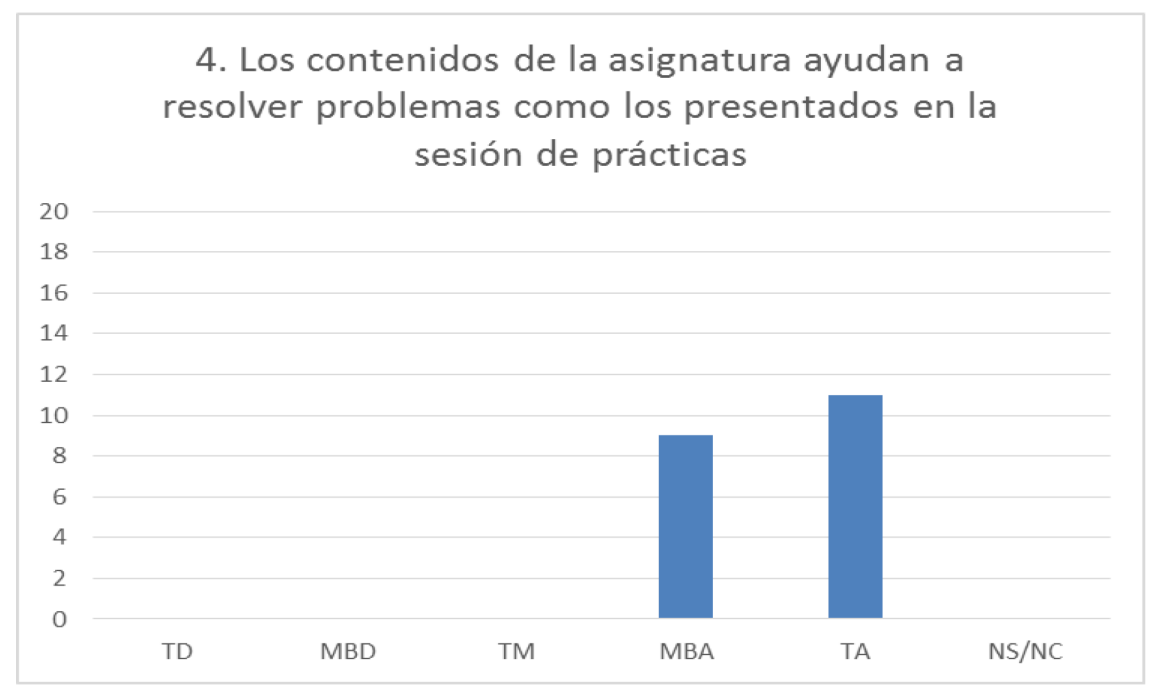

Fig. 6 Respuestas a la cuarta pregunta del cuestionario de la Tabla 3.

\section{Conclusiones}

En esta ponencia se presenta la experiencia llevada a cabo en la asignatura Vibraciones Mecánicas, con el objeto de integrar la competencia transversal de "conocimiento de problemas contemporáneos".

La competencia transversal de "conocimiento de problemas contemporáneos" hace referencia a la necesidad de que los estudiantes comprendan cuestiones y valores políticos, sociales, legales y medioambientales.

Debido a la naturaleza de la asignatura, que está orientada a la resolución de problemas en ingeniería, a priori parece difícil conseguir la integración de esta competencia. Sin embargo, debido a que los problemas en ingeniería son cada vez más complejos, el trabajo de la competencia transversal puede contribuir a ensanchar el horizonte de los alumnos a la hora de afrontar problemas más complejos que salen del ámbito estrictamente ingenieril.

La experiencia se llevó a cabo en una sesión de prácticas de laboratorio. Se visualizó un video corto y se realizó un análisis de este documental empleando el método del caso.

Por un lado se realizó una evaluación del grado de adquisición de la competencia a nivel global, a partir de las respuestas que formulaban los alumnos. Por otro lado, y en relación con los objetivos de este trabajo, se pasó un cuestionario anónimo con el fin de evaluar la actividad. Los resultados de este cuestionario resultaron plenamente satisfactorios ya que indican que el trabajo de esta competencia contribuyen a mejorar el aprendizaje en la asignatura Vibraciones Mecánicas.

No obstante, y fruto de esta experiencia en la puesta en marcha de esta actividad para integrar la competencia CT-10 en esta asignatura, se podrían incorporar mejoras como la recogida de evidencias del trabajo de los alumnos reflejado en un portafolio o informe de la actividad o bien la grabación de la sesión en una sala habilitada para tal efecto. Con esto se 
Integración de la competencia transversal de "conocimiento de problemas contemporáneos" en la asignatura Vibraciones Mecánicas

persigue que el profesor esté involucrado en la propia actividad y la evaluación se pueda hacer a posteriori.

\section{Agradecimientos}

Este trabajo ha sido financiado por el Vicerrectorado de Estudios, Calidad y Acreditación y la Escuela Técnica Superior de Ingenieros Industriales (ETSII) de la Universitat Politècnica de València (UPV) a través de la convocatoria de ayudas para PIME 2015-2016, con el proyecto código B10.

\section{Referencias}

Universitat Politècnica de València, UPV (2015a). Portal de Competencias Transversales. $<\underline{\text { http://www.upv.es/contenidos/COMPTRAN/>[Consulta: } 4 \text { de noviembre 2015] }}$

TUNING PROJECT (2014). Approaches to teaching, learning and assessment in competences based degree programmes. $<$ http://www.unideusto.org/tuningeu $>$ [Consulta: 5 de abril de 2016]

VILLA, A. y POBLETE, M. (2007). Aprendizaje basado en competencias. Una propuesta para la evaluación de las competencias genéricas. Universidad de Deusto. Bilbao.

ANECA (2007) El profesional flexible en la Sociedad del Conocimiento. Madrid: Ministerio de Educación y Ciencia.

Universidad de Murcia, UM (2008). Competencias transversales de la Universidad de Murcia. $<$ http://www.um.es/documents/13951/87557/competencias.pdf $>$ [Consulta: 4 de noviembre 2015]

VILLA, A (2003). “Orientaciones Pedagógicas para la convergencia europea de educación superior”. En Seminario Internacional. Bilbao: Universidad de Deusto.

LEIVA-BRONDO, M., PEIRÓ, R., CEBOLLA-CORNEJO, J. and PÉREZ-DE-CASTRO, A.M. (2015). "Self and peer-assessment of knowledge of contemporary issues outcome in master degrees" en 7th International Conference on Education and New Learning Technologies. Barcelona, Spain, 6th-8th of July, 2015.

SCHWARTZ, J. D. and KRANOV A.A. (2012). "Introducing Contemporary Issues to Engineering Students: A Case Study Module”. En 2012 ASEE Annual Conference. $<$ http://www.asee.org/public/conferences/8/papers/3106/view $>$ [Consulta: 5 de abril de 2016] 
BALACHANDRAN, S., CLOUGH, J., JINKINS, P, and KILE, J. (2008). "Guidelines for integrating contemporary issues into engineering \& engineering technology curricula." En Proceedings of the 2008 ASEE North Midwest Sectional Conference. $<$ http://www.researchgate.net/publication/255608503_GUIDELINES_FOR_INTEGRATIN G_CONTEMPORARY_ISSUES_INTO_ENGINEERING_ENGINEERING_TECHNOL OGY_CURRICULA> [Consulta: 5 de abril de 2016]

SALA, A. L., SPENDLOVE, T. and RIDDELL, J. (2011). "Assessment of ABET program outcome J, "A Knowledge of contemporary issues". En 2011 ASEE Annual Conference \& Exposition. Vancuver, B. C. Canada. June 26-29, 2011.

$<$ http://www.asee.org/public/conferences/1/papers/883/view $>$ [Consulta: 5 de abril de 2016].

Universitat Politècnica de València, UPV (2015b). Rúbrica UPV CT-10. Conocimiento de problemas contemporáneos.

"London Millenium Brigde Opening". Youtube

$<\underline{\text { https://www.youtube.com/watch? }=\mathrm{gQK} 215720 \mathrm{SU}>}>$ [Consulta: 7 de septiembre de 2015] 